\title{
Drawing Architecture using Manga Techniques
}

\author{
$\mathrm{Y} . \mathrm{Qu}$ \\ Department of Computer Science and Engineering, The Chinese University of Hong Kong, Hong Kong \\ M.A. Schnabel \\ Department of Architecture, The Chinese University of Hong Kong, Hong Kong
}

ABSTRACT: Manga is a type of stylised bitonal drawings pleasing in terms of screen variety and tidy lines. In this paper, we present a method to draw architecture, spatial compositions and their details, including colours, textures, and tones, using manga drawing techniques. Given a colour image, such as photography, or drawing, our method finds the optimal solution for screening during the bi-tonal transformation, as well as a set of ideally abstracted line drawings. This offers a novel rendering appearance for the communication of architectural design. In our results we demonstrate successfully that our method generates manga-like drawings from an architectural image that preserve architectural key-elements, such as surface or material properties.

\section{INTRODUCTION}

Digital techniques introduced in the past decades have led a cultural shift in architectural design and its communication; new art forms and techniques influence the expression of architects' thoughts and ideas. However, continuing emphasis remains that hand-drawn sketches allow for a deeper understanding of design, which computer generated visualisations do not offer. Inevitably media play influential roles in the expression and communication of design. Subsequently the act of sketching allows for its own experience and interpretation of spatial arrangements (Goldschmidt 1991). Yet digitally aided sketching merges both realms into a novel expression of architectural communication, which we present in this paper.

Manga, a popular Japanese comic art form and medium of entertainment, is increasingly becoming known around the world. The graphical quality is unique in its elegant use of rich set of screens, and tidy and fine drawing styles. This novel way of visual communication of manga provides another realm for representing architectural drawings or photography, being real or virtual. Although the manga drawings depends on pure black and white (b/w) patterns and lines, or is bi-tonal represented, it is still possible to perceive the variety of textures or materials of the surfaces of buildings. During the screening process manga artists lay multifarious screens to express different semantics. Here the 'screening' refers to the process of laying pre-printed $\mathrm{b} / \mathrm{w}$ patterns over a certain area of the image. Screens are selected not solely according to the shading, or tone, but also ac- cording to texture, material property, chromaticity or even function of the underlying architecture that is being depicted and expressed.

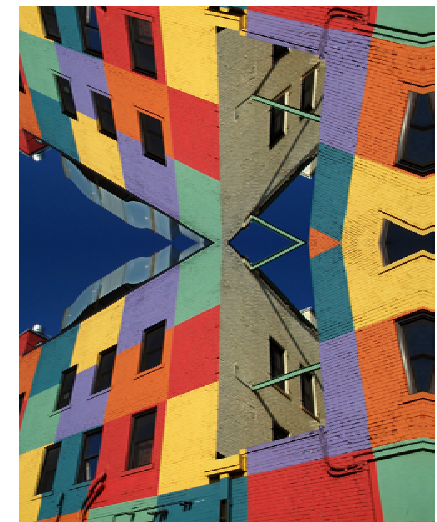

(a)

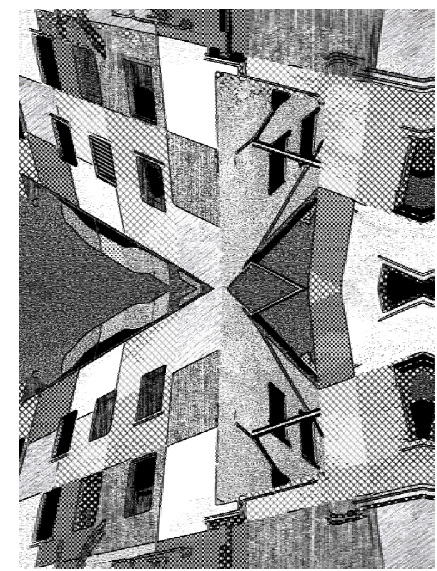

(c)

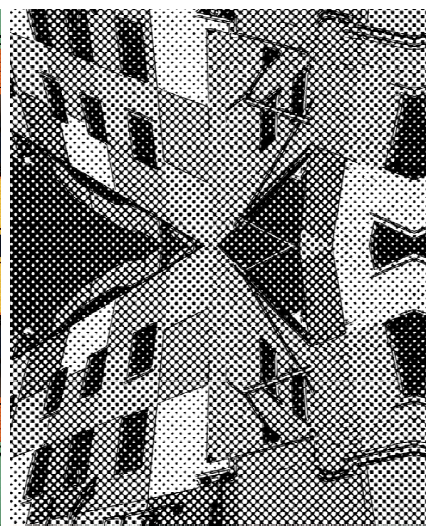

(b)

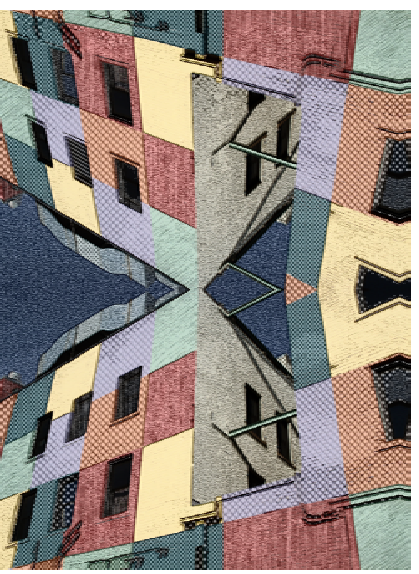

(d)
Figure 1. Generation of manga background from a real photograph (a). Commercial software (b) adopts halftone technique and present uniform screening result. Our results (c) \& (d) preserves colour distinguishability, texture similarity, and tone. 
Such a screening process is a very labour intensive undertaking. Once an architectural design is rich in details and elaborated in a high level, the process of creating a manga becomes even more complex. Speeding up the production some artists already employ computer techniques to convert images to manga backgrounds, using techniques such as 'half-toning' or 'hatching' (Figure 1a \& 1b). However, these digital methods produce only monotonous patterns and unsatisfactory results that are not in line with the rich expression of manga graphics and depicted architecture.

In this paper, we introduce a novel application that provides the user with an automatic screening tool, which is useful in architectural representation. Given an arbitrary colour image of an architectural arrangement, our novel computational method can instantly transfer the input into a bi-tonal multiscreening manga representation.

Our technique can deal with an arbitrarily complex image or colour palette. Rich sets of patterns/screens are employed to represent the original image with the goal of preserving three key-factors of manga graphical representation: 'chromaticity distinguishability', 'texture similarity', and 'tone similarity'. While the tone is preserved by matching the density of tone with the one of each pattern, our core contributions of our application are the preservation of chromaticity distinguishability and texture similarity. We develop a novel colour-to-pattern mapping based on the Multi-Dimensional Scaling (MDS) technique. MDS enables the relative distance to preserve the mapping from a '24 dimensional texture feature space' (of the screen patterns) to the 'two-dimensional chromaticity space'. As shown in Figure 1d, our results not only preserve the original tone, but also differentiate the chromaticity of the original image. Our system allows designers to interactively control and fine-tune their results.

\section{RELATED WORK}

\subsection{Architectural Sketch}

There is much written about manual or digital architectural sketches and the depiction of spatial arrangements using a two-dimensional medium. Since the $16^{\text {th }}$ century the tradition of drawing as an act of designing freed the architect from working on site at a scale of 1:1 and subsequently design became also an intellectual discipline engaged in the plane of paper. Goldschmidt (1991) argues that the architectural sketch is a mode of visual thinking and communication, which is crucial to a conceptual framework of the depicted architectural design. Despite the advancement of digital media, there remains a certain quality of a hand drawing and the architectural sketch remains the predominant medium of design- ing, communicating and construing. It incorporates abstraction, fuzziness as well as clarity and sharpness. As that the expressions of lines and hatches themselves become the objects of meaning and interpretation. A sketch explores from overall relationships to fine resolution of detailed attributes of the depicted design. In order to make the communication of space more precise the drawing makes use of graphic modifiers to convey information that go beyond the pure outlines or dimensions of the spatial arrangement (Robbins 1994).

\subsection{Bitonal Image Generation}

A straightforward way to produce computational bitonal images from greyscale/colour photographs is halftoning (Figure 1c). It exploits the spatial integration of human vision to approximate the intensity over a small local region with only b/w pixels (Knuth 1987, Floyd \& Steinberg 1974, Ulichney 1987, Jarvis et al. 1976). As artefact patterns may appear, some techniques (Velho \& Gomes 1991, Naiman \& Lam 1996) try to reduce the artefact patterns by adjusting the scanning path over the image. To preserve the edges during halftoning, Knuth (1987) enhances edges in a pre-processing step. Velho \& Gomes (1995) and Buchanan \& Verevka (1995) approximate edge regions by rearranging the clusters around edges. Recently, Pang et al. (2008) preserve the fine structure by optimizing the structure similarity.

Hatching is another technique to produce bitonal images (Figure 1b). Winkenbach et al. (1994) generated pen-and-ink illustrations by rendering a geometric scene with prioritized stroke textures. A userspecified "detail indication" can be used to highlight complex textures and minimize clutter. Salisbury et al. (1997) use direction fields to guide the orientation of strokes. Durand et al. (2001) suggest a thresholding model of strokes that can express a rich set of stroke styles to assist artists in achieving various styles. Grabli et al. (2004) improve the clutter control of lines by estimating the line density. This predicts the visual complexity before rendering and allows simplification.

Images generated by halftoning and hatching, containing more or less uniform patterns, may appear monotonous to the reader; traditional manga instead uses a wide variety of screens to enrich the viewing experience. Qu et al. (2008) proposed a novel richness-preserving manga screening method utilizes the variety of pattern to preserve the chromaticity distinguishability as well as the tone and texture similarities. Based on their work on pure screening part, we propose a complete system to generate manga style architectural drawings including screening, line drawings, and the final rendering. 
1.

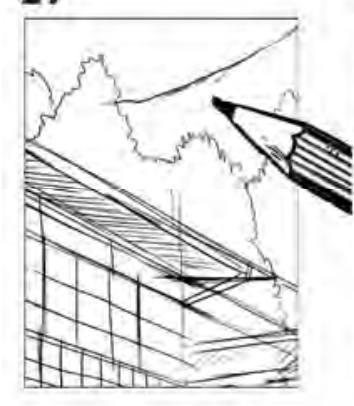

2.

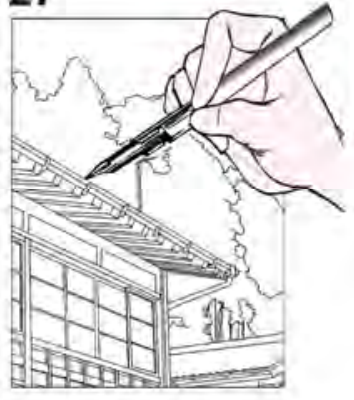

3.

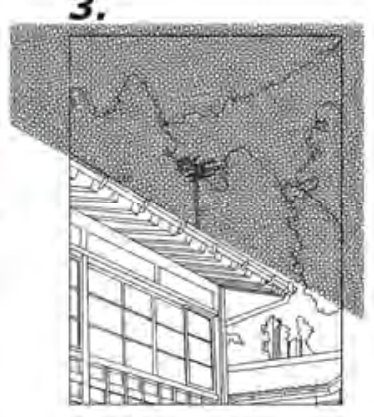

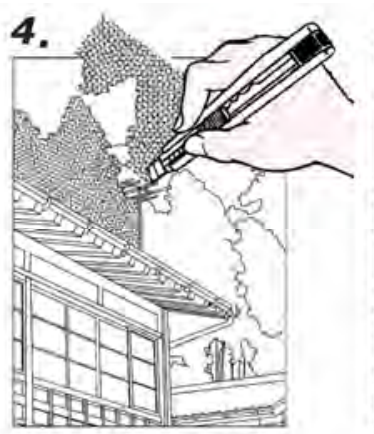

5.

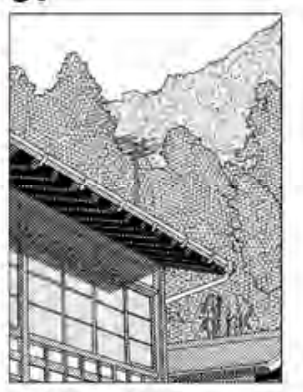

Figure 2. The typical workflow of drawing a manga frame

\section{CONVENTIONAL DRAWING SCHEMES}

\subsection{Traditional Manga Production}

The notion of manga techniques in this work is based on the schemes used in traditional manga production (Nagatomo 2003), which is widely adopted in manga creative industries. Figure 2 illustrates the typical five drawing steps for producing one mange image-frame. First, artists decide the perspective and roughly sketch the major structure of the scene with pencils (Step 1). Next, they finalize the drawing with ink (Step 2). With the precise lines in place, artists then begin the screening procedure. Based on the inked lines, manga artists usually select appropriate pre-print screen sheets to fill regions in order to express shading, tone, texture, or atmosphere. The selected screen paper, which is semi-transparent with pre-printed patterns, is then overlaid on each of the target regions (Step 3). The artist usually uses a knife to carefully carve out screen paper along the boundary and paste it on the target region (Step 4). The manuscript is ready for print when all the regions are overlaid with selected screens (Step 5). The task is rather tedious, time- and labourintensive, especially when large amount of irregular regions exist.

\subsection{Screen Library}

A traditional screen paper is a transparent layer of film, with a printed pattern on one side and adhesive on the back. Screen papers are produced by different companies in a variety of patterns and tones. Our digital screens consist of repeated patterns generated by texture synthesis methods as well as scanned paper screens. In the screen library, each type of screen (e.g. dot or hatch) has a range of density (tones) as shown in Figure 3. Note that the bitonal screens offer not only the one-dimensional tone density, but also the variety of patterns. In other words, the screen space is two-dimensional in nature.

\subsection{Conventional Architectural Screens}

Drafting is a universal convention that uses screens, symbols, hatches, line-types, -styles and widths to describe elements of a design, its properties, functions or materials. It is filled with represented knowledge and key information for a specific spatial composition. In addition to that, Architectural drafting is flexible and allows the creativity of the individual architect to influence the overall style of the screens and their variations. Mechanical engineering and Computer Aided Architectural Drafting (CAAD) typically have no personal style and depict neutral and standard screens following norms of the architectural, engineering and construction (AEC) industries. These drawing styles are defined e.g. in ISO 128 (2002).

The architectural sketch however, to which we refer here in this paper, does not employ these conventions. Similarly to an artistic drawing, various graphical elements and drawing techniques express visual clues of the depicted scene. Schön and Wiggins (1992) describe the architectural drawing as a 'reflective conversation with materials of a design situation'. 'A designer sees, moves and sees again. Working in some visual medium the designer sees what is 'there' in some representation of a site, draws in relation to it, and sees what has been drawn, thereby informing further designing.'

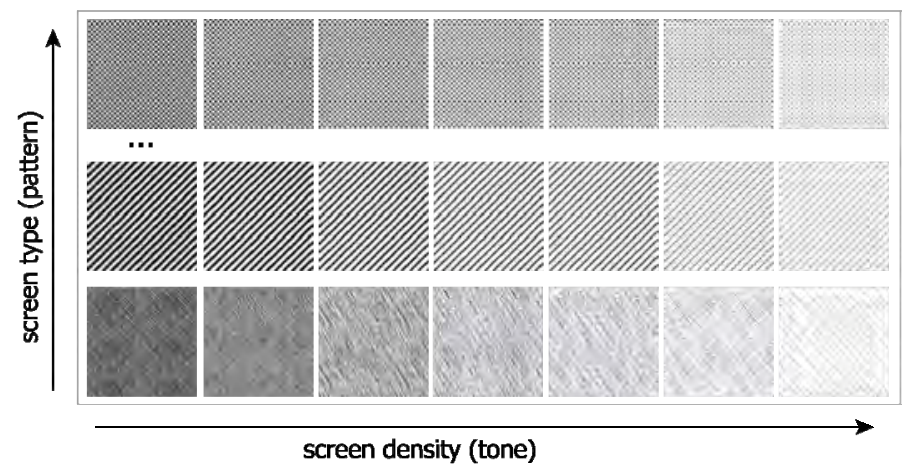

Figure 3. The screen space. Apart from tone variety, another dimension is the texture or pattern. 


\section{OVERVIEW OF THE MANGA DRAWING SYSTEM}

Richness preserving manga screening (Qu et al. 2008) offers a solution to generating bitonal manga screening for an input colour image. Their goal is to preserve the visual richness in the original photograph by utilizing not only screen density, but also the variety of screen patterns. Base on this work, we build a system to automatically draw architectural depictions using manga techniques. Our system consists of two major components, screening and line drawing, which are similar to the conventional manga production. For the line drawing part, we propose a line importance model to rank each line. With this ranking, architects can control the detail level of lines needed in their architectural manga, via a simple threshold. For the screening process, we utilize the solution of Qu et al. (2008) that automatically selects appropriate bitonal screens to represent (or fill up) different regions in the image. Our aim is to automate the screen selection process with the goal of richness preservation and style consistency to traditional manga.

Each type of screen supports a range of densities to represent different intensity. Therefore, whenever a screen is selected for a region, the intensity of this region can be approximated by matching the screen density. The major difficulty is to select an appropriate type of screen for each region, which can preserve the texture similarity and the colour distinguishability of the target regions in order to preserve the architectural content.

The system starts by segmenting the image into regions (Section 5.1). Then, the system can intelligently provide the optimal assignment of screens for different regions (Section 5.2). This is done by first projecting the available patterns from highdimensional texture feature space to the low dimensional colour space, and then optimizing texture similarity. In addition, users are still able to control and/or override the selection of screens via simply tuning a few parameters. Finally lines are detected and overlaid on the screens to finish the result (Section 6).

\section{SCREENING}

Screening is a technique for applying textures and shades to drawings, used as an alternative to hatching. In the conventional process, patterns are transferred to paper from pre-printed sheets, but the technique is also simulated in computer graphics.

\subsection{Segmentation}

As screens are laid over regions rather than individual pixels, we first identify regions, or segments, in the input reference image. Previous image segmentation methods have used various schemes to determine the partitioning in a digital image, so that each of the pixels in a region are similar with respect to some characteristic or computed property, such as colour, intensity, or texture. We perform the segmentation using mean-shift, which is a nonparametric clustering technique based on the analysis of a complex multi-modal feature space and delineation of arbitrarily shaped clusters (Comaniciu \& Meer 2002). With a kernel measure of the distance between pixels, it robustly produces noise-reduced segments. The meanshift segmentation result is usually over-segmented, which is obviously too fragmented for direct screening. Thus, we re-group segments according to their colour difference and proximity. Near segments with similar colours are grouped first. Each segment is then referred as the basic unit of the screen matching (Figure 4).

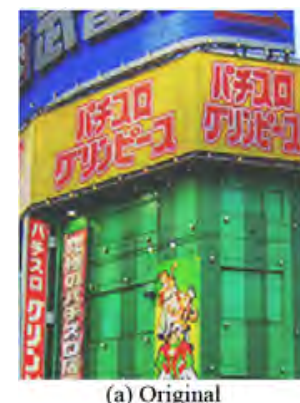

(a) Original

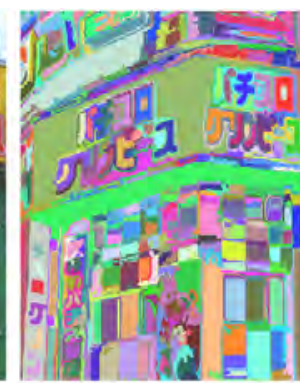

(b) Segmentation map

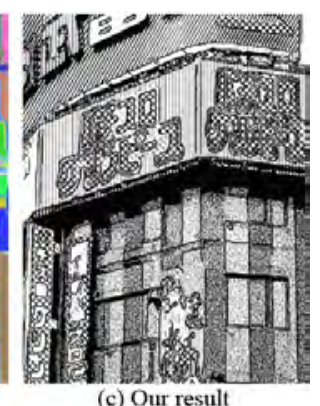

(c) Our result
Figure 4. (a) an image with rich content will have thousands of segments. (b) Semi-automatic segmentation plus manual screen assignment. (c) Our automatic screen assignment significantly relieves user intervention producing reasonable results.

\subsection{Screen Matching}

After segmentation, each segment is matched with an appropriate screen from the screen library. Each type of screen (e.g. dot or hatch) in this library has a range of density (tones) as shown in Figure 2 above. The screens are organized in a two-dimensional matrix with columns of varying density and rows of varying screen type.

The main objective of screen matching is to preserve the rich content of the original reference image. Here, we aim to preserve three classes of contents including tone (or luminance), texture and chromaticity distinguish-ability. Referring to the two available dimensions in the screen space (Figure 2), tone can be straightforwardly matched with the horizontal screen density axis. However, matching the tone alone does not produce satisfactory results as demonstrated in Figure 6b. This screening result ignores texture and chromaticity, and more importantly, its style is not consistent with the artworks of professional manga artists. Although the screening style of artists varies, they all enrich the drawing by introducing appropriate pattern varieties. 


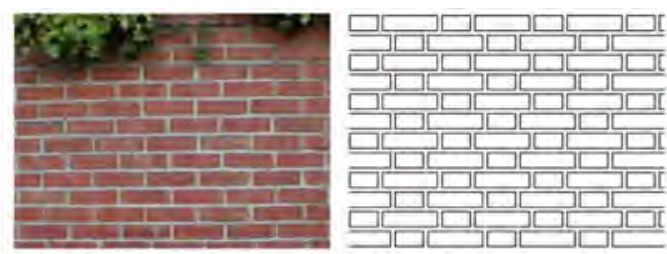

(a) Texture-based matching

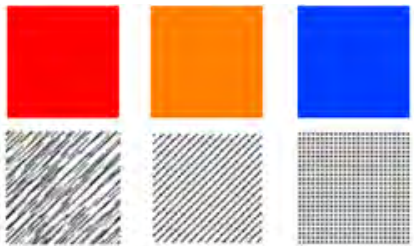

(b) Color-to-pattern mapping

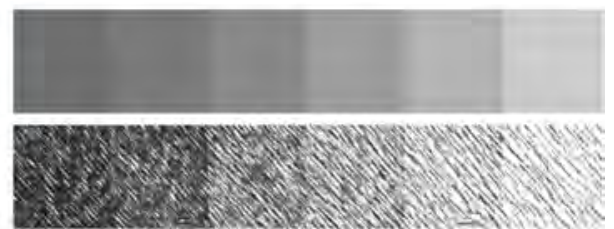

(c) Tone matching

Figure 5. Objectives of the screen matching: (a) Texture preservation, (b) chromaticity distinguishability, and (c) tone preservation. In (b), we assign the red and orange regions with two close patterns in below, as they are closer in colour; on the other hand, the blue region will be screened with a substantially different pattern.

The screening method consists of the following three steps:

- Texture-based matching. Segments containing apparent texture are first matched with screen patterns based on the texture similarity, as shown in Figure 5a.

- Colour-to-pattern mapping. Unmatched segments are then mapped to different pattern types with the goal of maintaining colour distinguishability, via a colour-to-pattern mapping. As shown in Figure 5b, suppose we are assigning the bottom patterns to the above three colours. It is desirable to assign the red and orange regions with two close patterns, as they are closer in colour. On the other hand, the blue region will be screened with a substantially different pattern.

- Tone matching. Finally, segments assigned to pattern types are screened by matching the tone. An illustration is shown in Figure 5c.

\subsubsection{Texture-based matching}

During the screen mapping, we give high priority to the texture feature preservation. That means segments exhibiting apparent texture characteristics are first assigned a screen pattern based on texture similarity. And such assignment will not be modified during the rest of the screening procedure.

To quantify the texture characteristics, we compute the texture features on both segments and screens using Gabor wavelets (Manjunath \& Ma 1996). It has been demonstrated as an effective texture identification technique for manga screens (Qu et al. 2006). Consider an image we compute its Gabor wavelet feature in a per-pixel manner. In other words, each Gabor wavelet feature is represented as a 24-dimensional vector.

For each segment in the reference and each screen in the screen library, we compute their average Gabor wavelet features as the representative. The matching can be done by measuring the Euclidean distance of the representative texture feature vectors between the segment and the candidate screen. Figure 5c shows an example matching of the textured roof with the screen "grid". Segments without such a proper match either contain no apparent texture characteristics or no suitable screen available in the library. Hence, they are left to the following colour-to-pattern mapping.

\subsubsection{Colour-to-Screen Mapping}

After the above texture-based matching, only textural regions are assigned with screens for the sake of texture preservation. There are still many segments may still not be assigned with any screen due to their textureless characteristics. In this section, we assign these non-textural regions according to their chromaticity difference among segments.

The solution proposed for chromaticity distiguishability is to maintain the perceived distances among different segment colours during the screen type assignment. In other words, we want to maintain the same distance relationship among different colour segments after mapping to the screen space.

The problem is that the screen feature is calculated in a high-dimensional space (24D), while the colour is in a low-dimensional space (2D). And there is no direct mapping between the different dimensional spaces. Note that we adopt the 'LAB' colour space that consists of one luminance $(\mathrm{L})$ and two chrominance channels (AB). Only the twodimensional chrominance is considered as the colour feature during the screen type assignment. As mentioned before, the luminance (tone) can be easily matched by selecting the appropriate screen density, once the screen type is selected.

We employ MDS (Cox \& Cox 1994) to maintain such distance relationship during the assignment of high dimensional screens to the low dimensional colours. The MDS reduces the dimensionality of the $24 \mathrm{D}$ texture feature to $2 \mathrm{D}$, so that we can do the mapping of texture to colour in the 2D space (Figure $6 b)$. This visual richness preserving screen assignment is called colour-to-screen mapping.

If we have computed the MDS for all screens, then we process each segment by first computing its average colour in the segment and then match a screen in the colour space. Note that the total number of distinct segment colours (in the magnitude of hundreds) is usually much larger than the total number of screen types used for a single manga. Manga artists seldom use hundreds of types of screen in one artwork. To maximize the usage of the available screens, we first perform the means clustering on the segment colours. The centroid of each cluster is used as a representative colour to match with the nearest screen coordinate in colour space. 


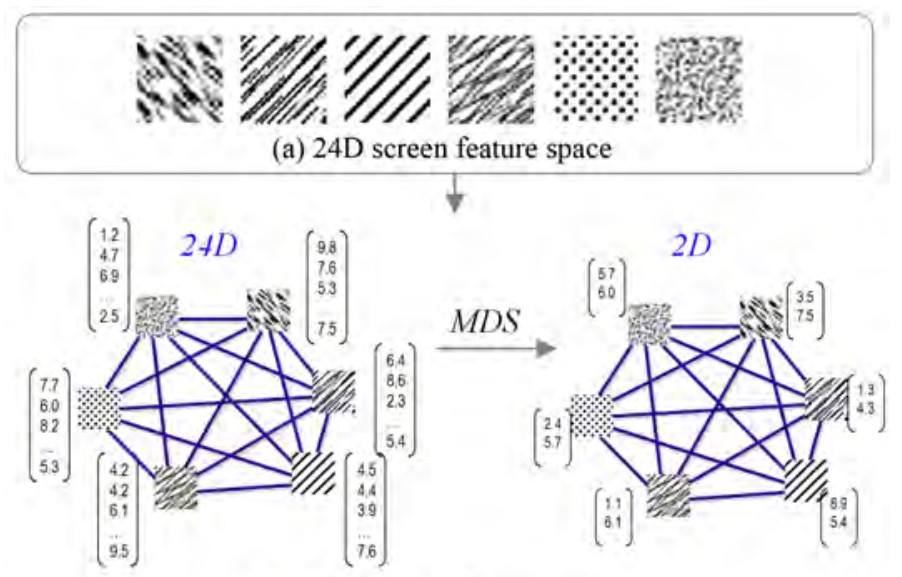

(b) The relative distance mainting dimension reduction from $24 \mathrm{D}$ to $2 \mathrm{D}$ by $\mathrm{MDS}$

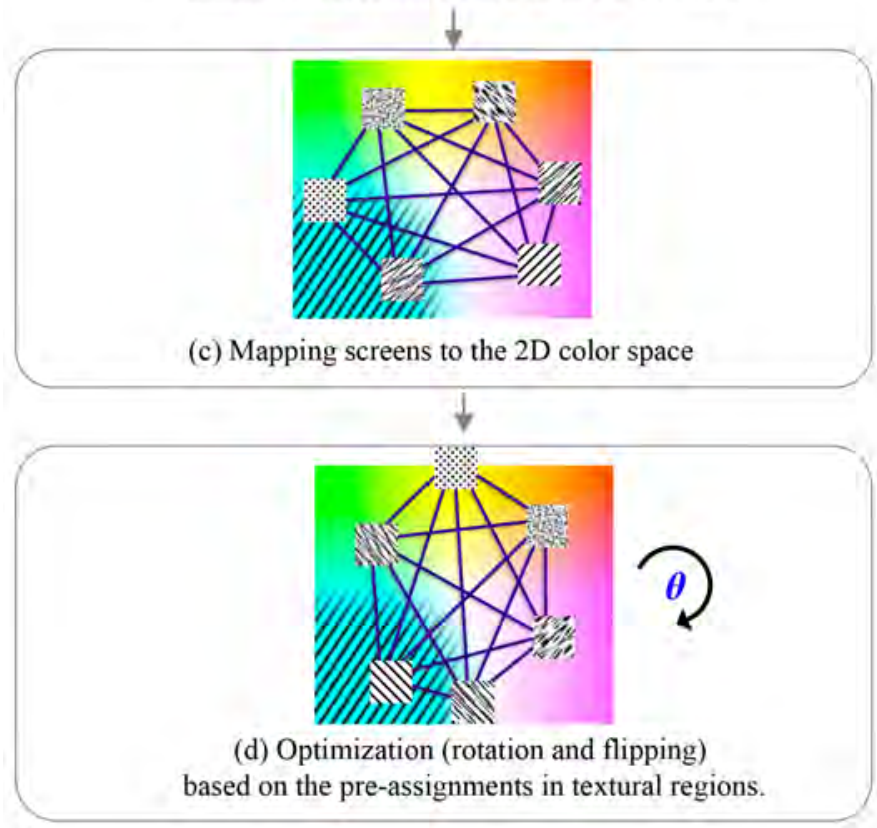

Figure 6. The MDS reduce the 24D texture feature into 2D, while maintaining the relative distance, so that the $24 \mathrm{D}$ textures can be mapped into 2D colour space.

Notice that such mapping is not unique, as the MDS only maintains the relative distances. We can still transform the mapped coordinates of the colour space by rotation, scaling, translation, and flipping. Both scaling and translation can be excluded as the coordinates segment colours can be normalized and zero-meaned. However, there are still two degrees of freedom left. To determine the best transformation, we actually perform colour-to-screen mapping on all segments including those already assigned with screens in the previous texture-based matching (Figure 6d), the cyan colour in the underlying colour space is pre-assigned with the screen "line". The rationale is that texture-based matching does not account for colours and, more importantly, we can utilize these pre-assigned segments to anchor the transformation. Hence, we optimize the transformation with an objective of maximizing texture similarity of the screens and segments. Figure $6 c$ shows the initial transformation with the cyan colour being assigned with a "cross hatching" pattern which obviously does not match well with the texture character- istic of the cyan region. By maximizing the texture similarity during adjustment of transformation, a better screen can be matched.

Our target of optimization is the minimal texture distance between the pre-assigned screens and the new assignments. For the purpose of relative distance preservation, only rotation and flipping are permitted. In specific, we quantize the rotation to 359 intervals, then the total number of possible transformation is only 718 (359 x 2). Even an exhaustive search can solve the optimization in seconds. Note that it is possible that the screens selected via the above optimization do not completely coincide with the pre-assigned screens from the first stage. In that case, we simply use the pre-assigned screens, as we give higher priority to the texture similarity.

\subsubsection{Tone Matching}

After the first two stages, each segment has been assigned a screen type. The final step is to perform a tone matching by selecting the appropriate screen density. We match the overall greyness of the screen with the average luminance of the segment. Next, we need to rotate the chosen screen (with specific density) in order to align with the texture characteristics in the segment. Note that the Gabor wavelet texture feature we employed is rotational invariant. Such matching is performed on each segment by the one by one manner until all regions are processed.

\section{LINE DRAWING AND ABSTRACTION}

The lines drawn by manga artists are usually tidy without being too crowded or chaotic. They intentionally omit many detail lines in order to maintain the tidiness of the manga. However, structural lines are retained, meaning that some lines are more important than others, and these lines usually form the main structure of a building or spatial arrangement.

Edge detection extracts lines purely based on the intensity gradient in the image. No attention is paid to the importance of a line. In many cases, edges obtained by automatic edge detection methods are far from satisfactory as demonstrated in Figure 7b.

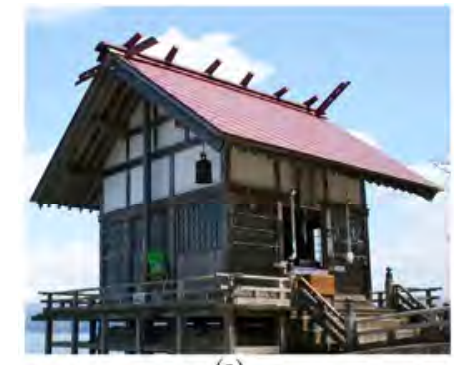

(a)

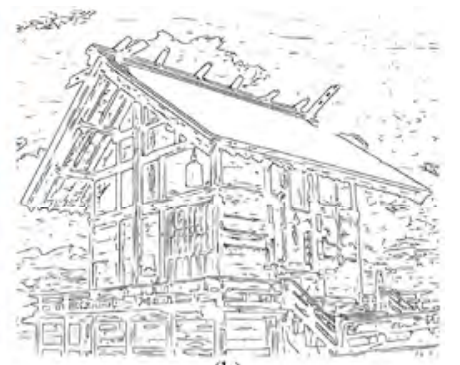

(b)
Figure 7. Edge detection result (Meer \& Georgescu 2001): (a) Original image (b) Edge detection result 
These noisy edges cannot be easily eliminated by parameter tuning of the edge detection, as important structural lines may also be removed indifferently during the tuning. Therefore, we propose a model to measure the importance of a line and unimportant lines are filtered out by adjusting a single threshold.

\subsection{Line Formation}

We begin our line formation from the edge detection on the original image. We adopt an improved edge detection method (Meer \& Georgescu 2001), which shows the ability to detect consistent results, even on weak edges. The detected edge pixels however are only a set of unstructured entities, so we first connect them using 8-connectivity. To resemble lines drawn by the artist, which usually contains no branching or self-intersection, we break the lines into pieces at the point with abrupt change in tangent. We refer the result as line map.

\subsection{Line Importance Modelling}

To tell structural lines from details, the key is to determine the importance of a line, so that a high importance value indicates structural lines while lower value indicates detail lines.

Architects usually first draw the long and straight structural lines during sketching. The short and curly lines are drawn at later stages or even omitted (Cheng 2006). Hence, the curliness and the length of lines suggest its importance. We also believe that lines associated with the major segment in the photograph should be more important. This suggests four factors are to be considered, the length, the curliness of a line, the size of largest associated segment, and the visibility value.

We measure the curliness by computing the deviation of curvatures of each edge pixel on the line. The curvature at each pixel can be estimated from the fitted polylines. The length can be obtained by counting the number of pixels on a line. The visibility level is computed by first forming an image pyramid, and lines with a counterpart in higher level have a larger visibility value. We identify the structural lines (lines associated with the major components in the photograph) by measuring also the size of its largest associated segment. The size of a segment can be obtained from our segment map (Section 4.2.1). The final line importance value will be decided by the overall curliness, length, visibility level, and the size of its largest associated segment.

Figure $7 \mathrm{~b}$ shows the unstructured edges from the raw edge detection result. With the proposed line importance model, we can generate the lines with a desired degree of abstraction to simulate manga style architectural drawings with different level of complexity or line abstraction. Figure 8 shows five different levels of abstraction of lines.

\section{RESULTS AND DISCUSSION}

Variety of input examples, including architectural photographs, colour drawings and artworks of spatial descriptions have been testified with our automatic manga style architectural drawing system. We have implemented the described system on a 2.6GHz Intel Core2 CPU computer with 3GB memory. The solution time for an $800 x 800$ image is only about two minutes, where the time includes segmentation, texture matching, colour-to-pattern mapping, tone matching, and the minimal user control.

In all the resulting images, we laid the same set of lines, determined in Section 5 above, over all the screen renderings compared in this paper, including the manga screening, which is determined in Section 4 , and the halftoning and hatching results, for the sake of fair comparison. As illustrated in Figure 9, the result (d) is generated by blending the screening result (b) with the line abstraction result (c). This technique allows communicating architectural design and features in a novel way, whereby thresholds and details can be emphasised and distinct elements highlighted. This is of particular interest in a context of design-learning or explanation of architectural intensions.

In Figure 10 we show an example that demonstrates the chromaticity distinguishability of the proposed system. Unlike the monotonous pattern appeared in the halftone result in Figure 10b, the colour variety in the input photo is well preserved by with screen variety as shown in $10 \mathrm{~d}$. We used five screens to represent five distinctive input colours (Figure 10c). By blending our result with the input image, such result illustrates the faithfulness of our textural presentation and emphasises the relationship of building elements with each other as well as to the whole architectural design.

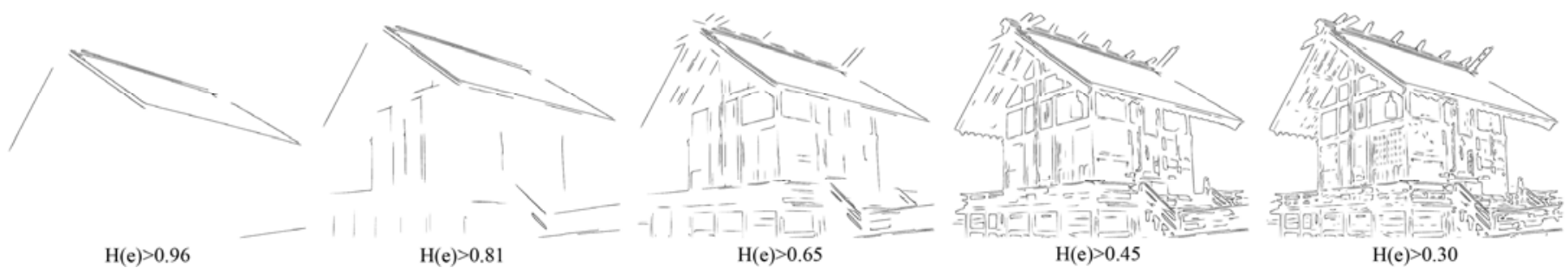

Figure 8. Level of line abstraction controlled by the line importance values. 


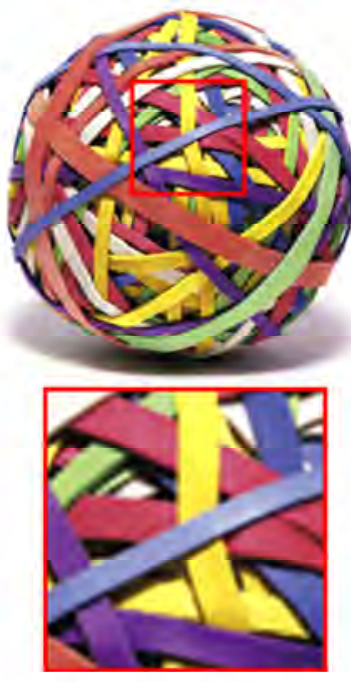

(a) Original image
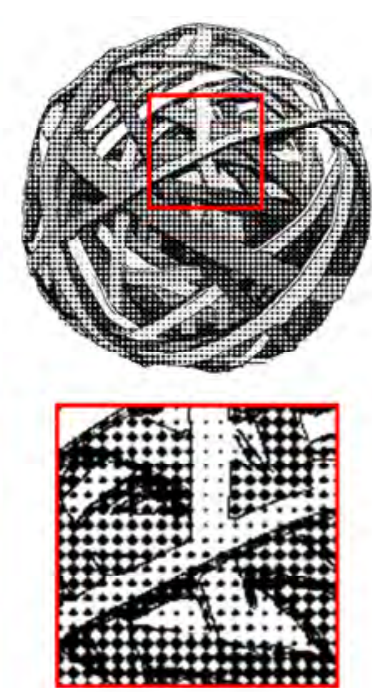

(b) Halftone result
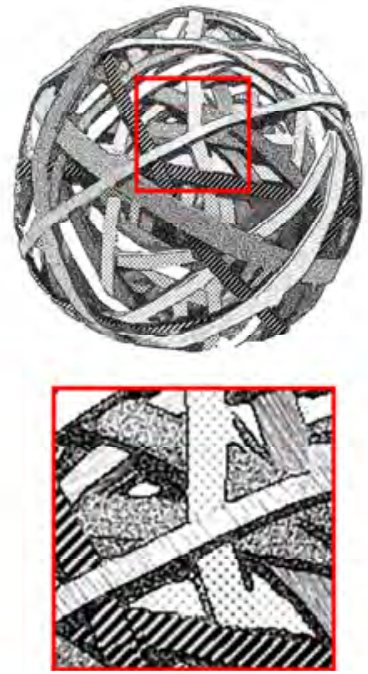

(c) Our result with various screen types
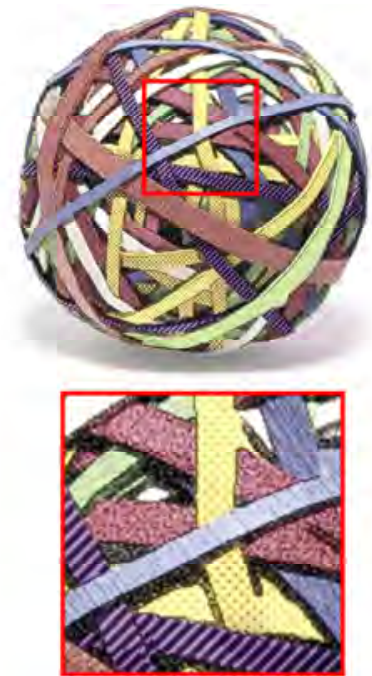

(d) Blending (c) with input photo for comparison

Figure 10. Unlike the monotonous pattern in halftone technique (b), the colour variety in a colour input photo or drawing will be well preserved with screens variety, as shown in (c), by our colour-to-pattern mapping (d).

This is particular useful to communicate large concepts that feature a variety architectural topologies, material, form, details as well as additional abstract information, such as design intent, light qualities, movement, and depth. Subsequently manga can enhance the communication of architectural representations. Our technique allows designers to abstract spatial relationships while at the same time preserve properties of material, surface or detail.

The narrative of a manga has long been defined by the figure-ground synergy since its conception that is the depiction of character or protagonist (figures) going through a certain action or event in a setting (ground). The idea of a spatial character starts by placing the emphasis onto seeing the background, the setting as the focus for narrative, with or without the figures as supporting actors ( $\mathrm{Ng}$ et al. 2006). Subsequently the protagonist in our drawings shifts from a character or person to the architecture itself.

Our technique allows for the development of a new gene of architectural design communication that reaches beyond graphical depiction of architecture.

Figures 1, 9, 11 and 12 demonstrate the effect of the global optimization, which considers all the texture preservation, chromaticity distinguishability, as well as tone preservation. During the bitonal process, images with large variety of colours appear more pleasing by our method, comparing with the start-of-the-art halftoning and hatching techniques.

The strengths of architectural drawings lay in the ambiguity and abstract translations of the original depicted spaces and designs. Our technique allows architects to blend from a photorealistic to an abstract depiction of architecture using a distinct expression of manga style visual communication.

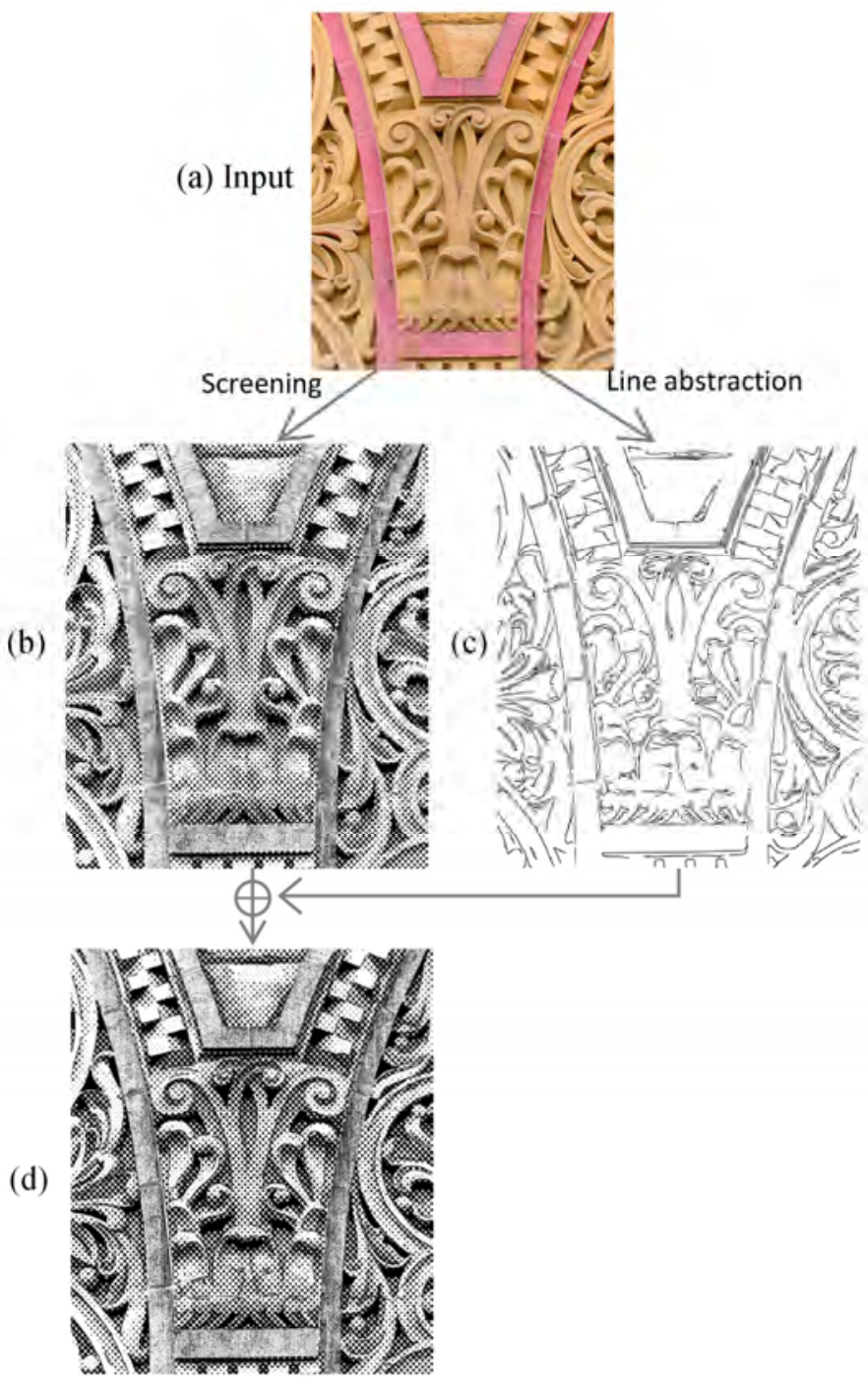

Figure 9. Architectural drawing using manga techniques. The result (d) is generated by blending screening result (b) with the line abstraction result (c). 


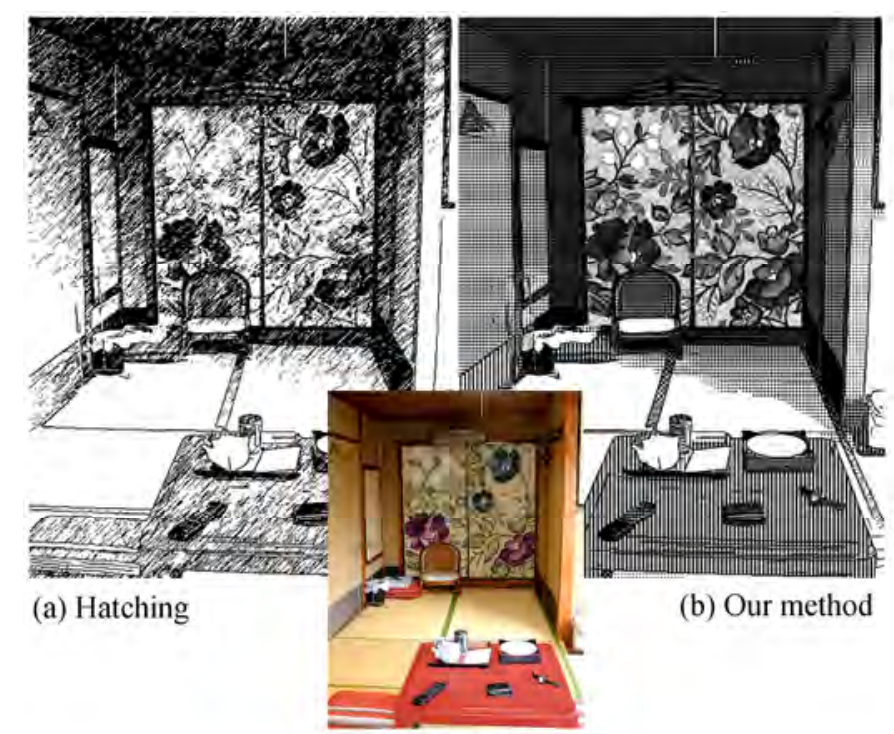

(c) Original

Figure 11. "Tea house”. Our result (b) properly preserves the multiple textures and colour distinguishability in the original photo (c).

\section{CONCLUSIONS}

We have presented a novel bi-tonal image drawing technique for architectural design that utilize manga drawing techniques, and provide an optimal screening scheme by using multiple screens and level-ofdetail line abstractions. The high dimensional screens enable the texture and tone preservation, as well as the chromaticity distinguishability of the depicted original. Our results give manga-like drawings comparable to those manually prepared by artists, and the system is efficient and convenient in use in architectural design and communication. Additionally we successfully demonstrated how the specific drawing technique of manga can be utilized to design and communicate spatial arrangements. The intersections of manual and digital instruments mirror the working styles of architects, who deal with a variety of realms, instruments and stakeholders. Hereby manga architectures aid not only users to understand larger architectural concepts, properties of form, material, light, etc, but aids in story telling and consultancy of professionals (Schnabel \& Howe 2010). Laypersons easily can access the architects' intension of design and function.

In future work, we would like to explore other options of colour spaces and texture spaces. Currently, we measure the chromaticity similarity in CIE L*a*b* space and pattern similarity in Gabor wavelet feature space. It is still an open problem to understand whether these two kinds of similarities are linear to our human perceptual distance. This will lead to a novel understanding of how architectural design is communicated and understood.

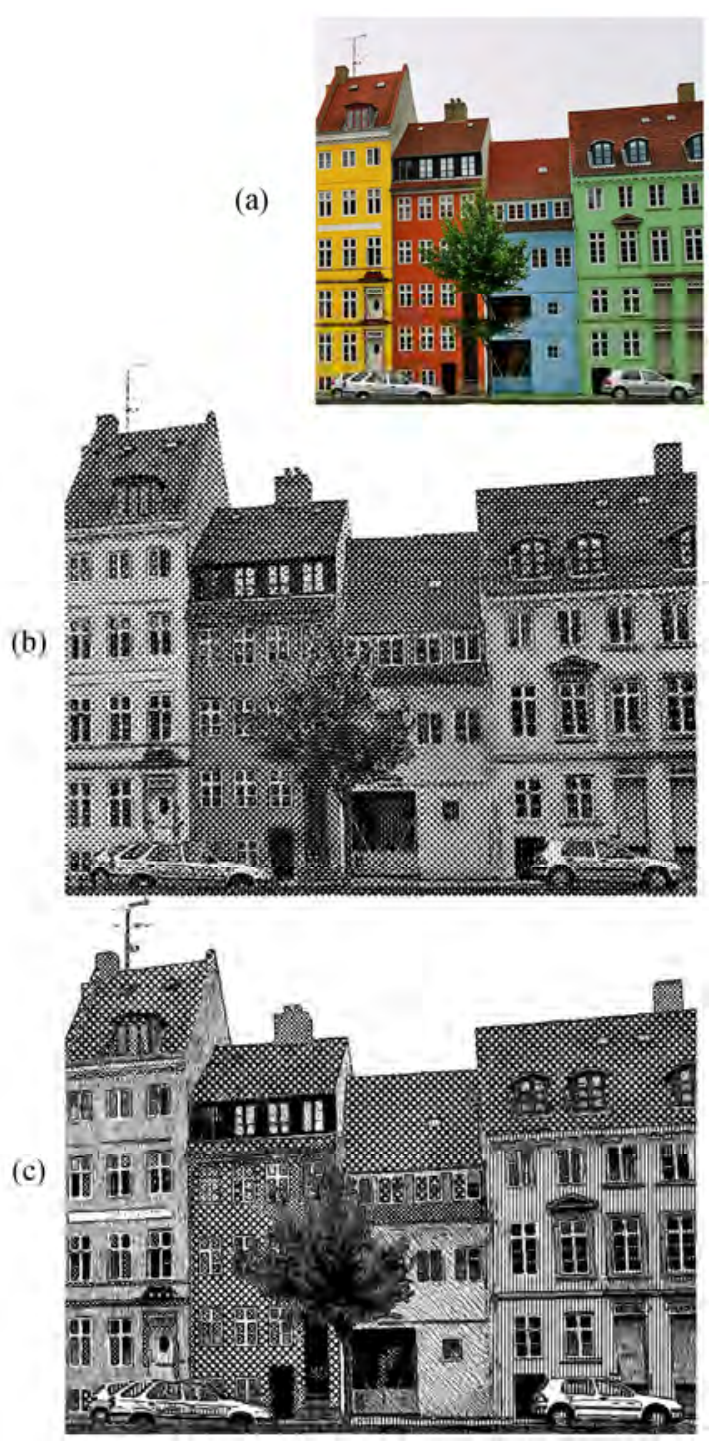

Figure 12. "Colour house”. (a) Original image, (b) halftone method, and (c) our method.

\section{REFERENCES}

Buchanan, J.W. \& Verevka, O. 1995. Edge preservation with space-filling curve halftoning. Proceedings of Graphics Interface 95: 75-82.

Cheng, N.Y.-W. 2006. Learning design sketching from animations and storyboards. International Journal of Architectural Computing 4(1): 1-17.

Comaniciu, D. \& Meer, P. 2002. Mean shift: A robust approach toward feature space analysis. IEEE Trans. Pattern Anal. Mach. Intell. 24(5), 603-619.

Cox, T.F. \& Cox M.A.A. 1994. Multidimensional Scaling. London, UK: Chapman \& Hall

Decarlo, D. \& Santella, A. 2002. Stylization and abstraction of photographs. SIGGRAPH '02: Proceedings of the 29th annual conference on Computer graphics and interactive techniques. New York, NY. ACM Press. 21: 769-776.

Durand, F., Ostromoukhov, V., Miller, M., Duranleau, F. \& Dorsey, J. 2001. Decoupling strokes and high-level attributes for interactive traditional drawing. Proceedings of the 12th Eurographics Workshop on Rendering Techniques, London, UK. Springer-Verlag: 71-82.

Floyd, R.W. \& Steinberg, L. 1974. An adaptive algorithm for spatial grey scale. SID International Symposium Digest of Technical Papers, Washington, D.C. Society for Information Display: 36-37. 
Goldschmidt, G. 1991. The Dialectics of Sketching. Creativity Research Journal. 4(2):123-143.

Gooch, A.A., Olsen, S. C., Tumblin, J. \& Gooch, B. 2005. Color2gray: saliencepreserving colour removal. ACM Trans. Graph. 24(3): 634-639.

Grabli, S., Durand, F. \& Sillion, F. 2004. Density measure for line-drawing simplification. Proceedings of Pacific Graphics.

ISO 128 (2002). Standards Handbook: Technical drawings Volume 1: Technical drawings in general.

Jarvis, J. F., Judice, C.N. \& Ninke, W.H. 1976. Survey of techniques for the display of continuous tone pictures on bilevel displays. Computer Graphics Image Process 5(1): 13-40.

Knuth, D.E. 1987. Digital halftones by dotdiffusion. ACM Transactions on Graphics 6(4): 245-273.

Li, Y., Sun, J., Tang, C.-K. \& Shum, H.-Y. 2004. Lazy snapping. ACM Transaction on Graphics 23(3): 303-308.

Manjunath, B.S. \& Ma, W.Y. 1996. Texture features for browsing and retrieval of image data. IEEE Transactions on Pattern Analysis and Machine Intelligence 18(8): 837842.

Mantiuk, R., Myszkowski, K. \& Seidel, H.-P. 2006. A perceptual framework for contrast processing of high dynamic range images. ACM Trans. Appl. Percept. 3(3): 286-308.

Meer, P. \& Georgescu, B. 2001. Edge detection with embedded confidence. IEEE Transactions on Pattern Analysis and Machine Intelligence 23(12): 1351-1365.

Nagatomo, H. 2003. Draw Your Own Manga: All the Basics. Japan: Kodansha International Ltd.

Naiman, A. \& Lam, D. 1996. Error diffusion: wavefront traversal and contrast considerations. Proceedings of Graphics Interface 96: 78-86.

Ng, K, Schnabel, M.A. \& Kvan,T. 2006. Architectural Animation becomes Alive - Creating Spatial Narrative with Spatial Characters for Animations, Communicating Space(s) Volos, Greece. eCAADe: 598-603
Pang, W.-M., Qu, Y., Wong, T.-T. Cohen-Or, D. \& Heng, P.A. 2008. Structure-aware halftoning. ACM Trans. Graph. 27(3): 1-8.

Qu, Y., Pang, W.-M., Wong, T.-T. \& Heng, P.-A. 2008. Richness-preserving manga screening. ACM Transactions on Graphics (SIGGRAPH Asia 2008 issue) 27: 155:1-155:8.

Qu, Y., Wong, T. T. \& Heng, P. A. 2006. Manga colorization. ACM Transactions on Graphics, SIGGRAPH 2006. 25: 1214-1220.

Robbins, E. 1994. Why Architects Draw. Cambridge, MA: MIT Press.

Salisbury, M.P., Wong, M.T., Hughes, J.F. \& Salesin, D.H. 1997. Orientable textures for image-based pen-and-ink illustration. SIGGRAPH '97: Proceedings of the 24th annual conference on Computer graphics and interactive techniques, New York, ACM Press/Addison-Wesley Publishing Co: 401- 406.

Schnabel, M.A. \& Howe, E.L.C. 2009. Client design literacy and consultancy skills in architectural education, N. Gu, M. Ostwald \& A. Williams (eds.), Computing, Cognition and Education - Recent Research in the Architectural Sciences. Newcastle, Australia: ANZAScA \& Uni. of Newcastle.

Schön, D.A. \& Wiggins, G. 1992. Kinds of seeing and their functions in designing. Design Studies. 13(2): 135-156.

Ulichney, R. A. 1987. Digital Halftoning. Cambridge, MA: MIT Press.

Velho, L. \& Gomes, J.M. 1991. Digital halftoning with space filling curves. Computer Graphics 25(4): 81-90.

Velho, L. \& Gomes, J.M. 1995. Stochastic screening dithering with adaptive clustering. Computer Graphics (Proceedings of SIGGRAPH' 95): 273-276.

Winkenbach, G. \& Salesin, D. H. 1994. Computer-generated pen-and-ink illustration. Proceedings of SIGGRAPH 1994:, 91-100. 